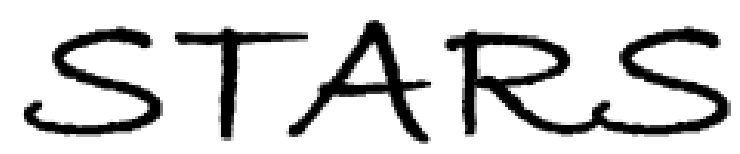

University of Central Florida

STARS

$1-1-2008$

\title{
Injection-seeded internal-reflection-mode p-Ge laser exceeds 10 W peak terahertz power
}

\author{
A. V. Muravjov \\ University of Central Florida \\ H. Saxena \\ University of Central Florida \\ R. E. Peale \\ University of Central Florida \\ C. J. Fredricksen \\ O. Edwards
}

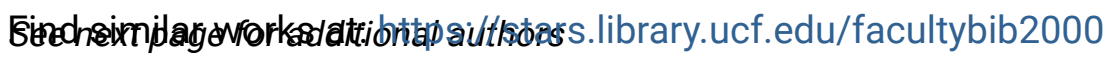

University of Central Florida Libraries http://library.ucf.edu

This Article is brought to you for free and open access by the Faculty Bibliography at STARS. It has been accepted for inclusion in Faculty Bibliography 2000s by an authorized administrator of STARS. For more information, please contactSTARS@ucf.edu.

\section{Recommended Citation}

Muravjov, A. V.; Saxena, H.; Peale, R. E.; Fredricksen, C. J.; Edwards, O.; and Shastin, V. N., "Injection-seeded internal-reflection-mode p-Ge laser exceeds $10 \mathrm{~W}$ peak terahertz power" (2008). Faculty Bibliography 2000s. 751.

https://stars.library.ucf.edu/facultybib2000/751

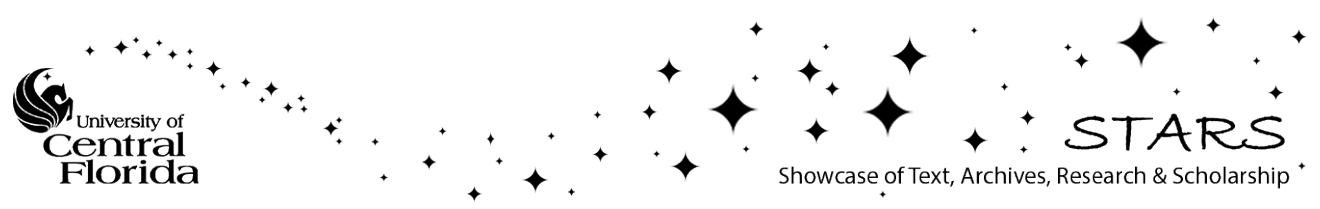




\section{Authors}

A. V. Muravjov, H. Saxena, R. E. Peale, C. J. Fredricksen, O. Edwards, and V. N. Shastin 


\section{Injection-seeded internal-reflection-mode $p$-Ge laser exceeds $10 \mathrm{~W}$ peak terahertz power}

Cite as: J. Appl. Phys. 103, 083112 (2008); https://doi.org/10.1063/1.2903140

Submitted: 21 December 2007 . Accepted: 27 January 2008 . Published Online: 22 April 2008

A. V. Muravjov, H. Saxena, R. E. Peale, C. J. Fredricksen, O. Edwards, and V. N. Shastin

\section{ARTICLES YOU MAY BE INTERESTED IN}

$186 \mathrm{~K}$ operation of terahertz quantum-cascade lasers based on a diagonal design Applied Physics Letters 94, 131105 (2009); https://doi.org/10.1063/1.3114418

Low-threshold terahertz quantum-cascade lasers

Applied Physics Letters 81, 1381 (2002); https://doi.org/10.1063/1.1498861

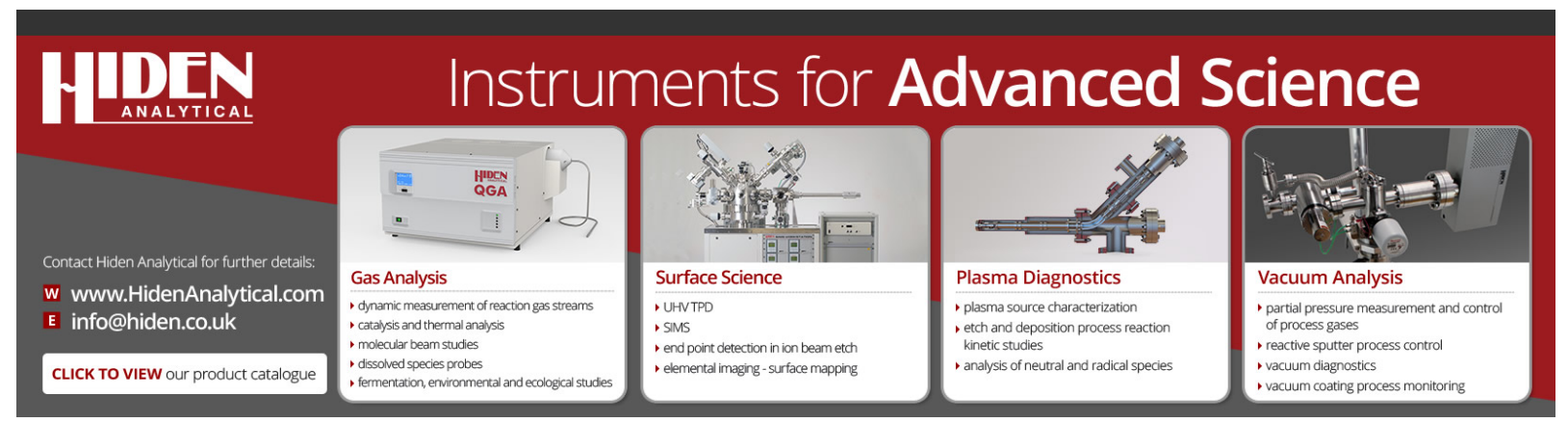




\title{
Injection-seeded internal-reflection-mode $p$-Ge laser exceeds $10 \mathrm{~W}$ peak terahertz power
}

\author{
A. V. Muravjov, ${ }^{1, a)}$ H. Saxena, ${ }^{1}$ R. E. Peale, ${ }^{1, b)}$ C. J. Fredricksen, ${ }^{2}$ O. Edwards, ${ }^{2}$ and \\ V. N. Shastin ${ }^{3}$ \\ ${ }_{1}^{1}$ Department of Physics, University of Central Florida, Orlando, Florida 32816, USA \\ ${ }^{2}$ Zyberwear, Inc., 2114 New Victor Rd., Ocoee, Florida 34761, USA \\ ${ }^{3}$ Institute Physics of Microstructures, Russian Academy of Sciences, GSP-105, Nizhny Novgorod 603600, \\ Russia
}

(Received 21 December 2007; accepted 27 January 2008; published online 22 April 2008)

\begin{abstract}
Injection seeding of a large active $p$-Ge laser crystal operating on total internal reflection modes is demonstrated with peak output power at the level of $40 \mathrm{~W}$ in the $1.5-4.2 \mathrm{THz}$ spectral range. The improvement over traditional $1 \mathrm{~W}$ axial mode $p$-Ge lasers is due both to spatially and temporally more efficient use of the available population inversion. (C) 2008 American Institute of Physics.
\end{abstract}

[DOI: 10.1063/1.2903140]

\section{INTRODUCTION}

The well-established inter-valence-band hot-hole laser in p-type germanium, tunable from 1.5 to $4.2 \mathrm{THz}$, is already known to generate coherent pulsed terahertz radiation at the level of $1 \mathrm{~W}$ peak power. ${ }^{1}$ In this paper we demonstrate the ability of the $p$-Ge laser system to generate pulsed terahertz radiation with an output power exceeding $10 \mathrm{~W}$. To optimize pumping efficiency and thermal management during the build-up time of the stimulated emission, we use a two-stage $p$-Ge laser system consisting of a large slave laser with a cavity operating on total internal reflection modes and with predelayed injection seed laser. The former maximizes the optical path and better fills the active volume. The latter reduces the usual laser build-up time and initiates oscillation in the slave laser when the active crystal is still cold and its gain is highest.

The theoretical limitation for the saturation intensity of stimulated emission within the active $p$-Ge laser crystals is $1-10 \mathrm{~kW} / \mathrm{cm}^{2} .^{2}$ In order to reach high output power densities at the typical small signal gain of $0.02-0.05 \mathrm{~cm}^{-1}$, the round-trip path must be at least several tens of centimeters. To provide long round-trip path in the active $p$-Ge laser crystal while maintaining reasonable crystal dimensions, we adopted a design based on total internal reflection from the lateral sides of the active crystal. ${ }^{3,4}$ Considering the high refractive index of $\mathrm{Ge}$ and the resulting rather steep angles of total reflection, a $10 \mathrm{~cm}$ long crystal operating on total reflection modes can provide an optical path length up to $80 \mathrm{~cm}$ per photon round-trip. Even though the total reflection mode in a rectangular rod-shaped active crystal has multiple self-crossings, it is significantly more efficient in filling the active volume than the usual axial mode cavity configuration.

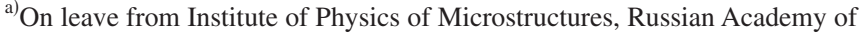
Sciences, GSP-105, Nizhny Novgorod 603600, Russia.

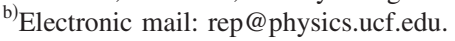

\section{EXPERIMENTAL DETAILS}

A large $66.75 \times 8.1 \times 6.15 \mathrm{~mm}^{3} p$-Ge crystal was used as a slave laser. The end faces and lateral sides of the crystal were polished parallel to each other with 30 arc sec accuracy, which allowed oscillation on internal reflection modes. The remaining lateral surfaces were prepared with evaporated and annealed aluminum Ohmic contacts coated with indium. The long axis was oriented along a 110 crystallographic direction. High-voltage pulses up to $3 \mathrm{kV} \times 1 \mathrm{kA}$ with $1-6 \mu \mathrm{s}$ duration were applied to the active crystal along the $1 \overline{1} 0$ crystallographic direction perpendicular to the long axis of the crystal using a thyratron-based pulser.

A small $p$-Ge laser with external copper mirrors and axial mode configuration was used as the seed laser. The active crystal was in the shape of a rectangular rod with dimensions of $18 \times 7 \times 5 \mathrm{~mm}^{3}$. The crystallographic orientations of the crystal were in the same sense as for the slave laser crystal. The small end faces were polished flat and parallel to within 30 arc sec. The lateral surfaces were ground flat but were left unpolished to prevent reflections. The output coupling mirror was a $3 \mathrm{~mm}$ diameter polished copper disk. The back mirror was a similar but larger copper disk covering the entire end face. Both mirrors were electrically isolated with $20 \mu \mathrm{m}$ sheet polytetrafluoroethylene (Teflon). Ion-implanted gold-coated Ohmic contacts were prepared on the $18 \times 5 \mathrm{~mm}^{2}$ faces of the active sample. An intracavity wavelength selector can be used to obtain high spectral pu-

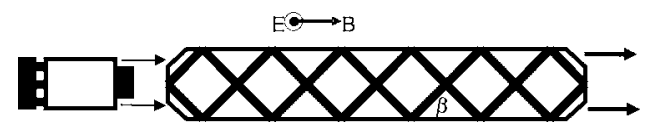

FIG. 1. Coupled laser scheme for high power terahertz generation. The large $p$-Ge slave laser operates on total reflection modes, which provides long round-trip path through the active crystal, required for high-power output. Collimated output of the radiation from the total internal reflection cavity is arranged through corner bevels. Similar bevels allow incoupling at the proper angle from the seed laser. The seed laser shown is a small independently excited $p$-Ge laser with small copper output mirror to allow outcoupling around the edges and (as an example) an intracavity lamellar grating wavelength selector at the large copper back mirror. 
rity of the seed laser emission, as it has been shown in our previous papers $^{5-9}$ and as is schematically indicated in Fig. 1, although we have not use it for these particular experiments. An insulated gate bipolar transistor pulse generator (Zaubertek) applied excitation pulses up to $1 \mathrm{kV} \times 400 \mathrm{~A}$ with $1 \mu$ s duration.

Figure 1 presents a schematic of the coupled laser system. Collimated output is achieved via polished bevels ${ }^{9}$ at the corners of the slave laser crystal. The seed laser injects radiation into the slave crystal via similar polished bevels such that a preferred internal reflection mode is excited. The optimum bevel angle was determined from the configuration of the strongest total internal reflection mode in the active crystal, which was determined from the fine structure of the laser emission spectrum. This spectrum was measured using a half-meter grating monochromator (Jarrell-Ash) with a 5 $\times 5 \mathrm{~cm}^{2}, 7.9$ lines $/ \mathrm{mm}$ grating blazed for $118 \mu \mathrm{m}$ wavelength (Newport). The spectrometer was calibrated using a far-infrared molecular gas laser (Coherent-DEOS SIFIR-50), and the best wavelength resolution was determined using this laser to be $0.3 \mu \mathrm{m}$, which equals the theoretical maximum for the grating used. From the spacing $\delta \nu$ of the spectral peaks (in wavenumbers), the propagation angle $\beta$ relative to the crystal axis (Fig. 1) is found according to ${ }^{3}$

$$
\delta \nu=1 /[2 n a \sin (\beta)]
$$

where $n=3.925$ is the refractive index of $\mathrm{Ge}$ at $4 \mathrm{~K}$ and $100 \mu \mathrm{m}$ wavelength and $a$ is the distance between lateral surfaces. Once the propagation angle is determined, the optimal bevel angle is found using the law of refraction, so that axial input and output radiation will selectively couple through the bevels to the laser mode propagating within the crystal at the angle $\beta$. To fabricate the bevels, the crystal was held in a machined jig at the proper angle, and $\sim 1 \mathrm{~mm}$ wide bevels were ground and polished on the $8.1 \mathrm{~mm}$ long edges.

Both seed and slave lasers were collinearly inserted within the bore of the same superconducting solenoid $(B$ $=0.3-2 \mathrm{~T}$ ), immersed in liquid helium at $4 \mathrm{~K}$, as it is shown in Fig. 1. The output of the seed laser was simply pointed at the back end of the slave laser from the distance of $\sim 1 \mathrm{~cm}$. No special provision (beyond the bevels) was made for input coupling. The radiation, for either laser independently operated or both together simultaneously operated, was measured in the beam just above the magnet by a Ge:Ga photoconductor within a brass light pipe. The same light pipe led the radiation out of the Dewar to a room temperature Golay cell (QMC) or to the spectrometer coupled with a $4 \mathrm{~K}$ silicon bolometer (Infrared Labs).

The laser emission was detected by a Ge photoconductive detector, of dimensions $4.3 \times 2.1 \times 6.0 \mathrm{~mm}^{2}$, doped to the level of $7 \times 10^{13} \mathrm{~cm}^{-3}$ with the shallow acceptor Ga having an ionization energy of $\sim 10 \mathrm{meV}$. The photoconductivity $\sigma$ of the detector at $4 \mathrm{~K}$, where carriers are bound to the acceptor centers, is proportional to the number of photoionized acceptors. The carrier relaxation time $\tau$ defines the integration time for accumulation of photocarriers, which for this doping concentration is of order 100 ns. An adjustable

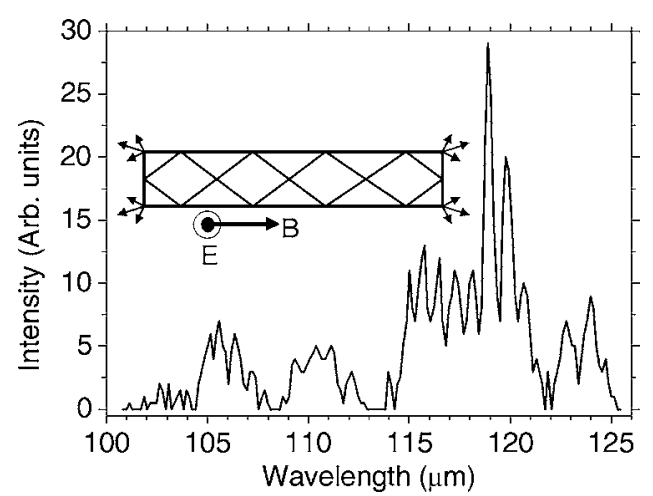

FIG. 2. Spectrum of the $p$-Ge slave laser operating on total internal reflection modes. The inset presents a schematic of the laser crystal with applied field vectors, showing operation on total internal reflection modes and nondirectional output coupling at the corners.

aperture on the front of the detector maintained a linear response, which allowed estimation of the absolute incident terahertz power according to

$$
P_{\text {collected }}=h \nu V \sigma /(e \mu \tau),
$$

where $h \nu$ is the photon energy, $\sigma$ is the measured photoinduced conductivity, $V$ is the volume of the detector, $e$ is the electron charge, and $\mu \sim 50000 \mathrm{~cm}^{2} / \mathrm{V} \mathrm{s}$ is a typical hole mobility for $\mathrm{Ge}$ at $4 \mathrm{~K}$. According to Eq. (2), the detector sensitivity was $2.2 \mathrm{~A} / \mathrm{W}$, which is in reasonable agreement with calibrated measurements of Ge:Ga detector sensitivity made in Ref. 10. The total emitted power of the laser is then found after multiplying $P_{\text {collected }}$ by a geometrical aperture factor and dividing by efficiency of incident radiation absorption in the detector. The latter factor is determined by estimating front surface reflection and the Ga acceptor photoionization cross section to have the value $\sim 1 / 3$ for our particular detector element.

\section{RESULTS}

Figure 2 presents a spectrum of the slave laser emission before bevels were made. In this mode of operation, laser radiation escapes the active crystal only due to scattering at the corners, as indicated in the inset. This uncollimated radiation is strongly attenuated through reflections on the inner surfaces of the light pipe. Nevertheless, the signal-after passing $1 \mathrm{~m}$ of light pipe, two $90 \mathrm{deg}$ bends, and a spectrometer-remains sufficiently strong to collect a good spectrum at the highest resolution. Analysis of the peak separation in the mode structure determines a propagation angle of the strongest mode inside the active crystal $\beta$ of $20.22 \mathrm{deg}$ with respect to the long crystal axis. This corresponds to four reflections on lateral surfaces per pass or ten reflections per photon round-trip, as indicated in the inset of Fig. 2. According to Snell's law, we determined an ideal bevel angle of $63.17 \mathrm{deg}$ as measured from the lateral surfaces. Such bevels were prepared, after which the output at the end of the light pipe was noted to have increased by a factor of $\sim 20$ due to the improved collimation of the output. Interestingly, after introducing the bevels, in addition to the previously dominant mode, another mode appeared in the spectrum having a propagation angle corresponding to the number of reflections 


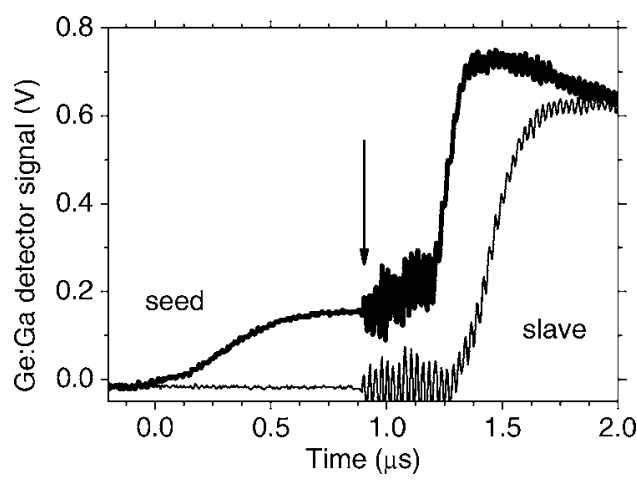

FIG. 3. Coupled laser emission recorded by a fast Ge:Ga photoconductor with (heavy trace) and without (light trace) injection seeding. The arrow indicates the beginning of the slave laser excitation, and the noise burst from electromagnetic interference persists while the slave laser excitation current ramps up to its optimum value.

per round trip increased by 2 , which was determined from changes in the fine structure of the emission spectrum.

Figure 3 presents time resolved measurements that show the effect of seeding in reducing the delay between excitation high-voltage pulse and development of the stimulated emission of the slave laser. Here, the laser signal is detected by a $4 \mathrm{~K} \mathrm{Ge}: \mathrm{Ga}$ detector for different delay times of the slave laser excitation relative to that of the seed laser. The arrow marks the slave laser excitation edge, and the noise burst from electromagnetic interference persists while the excitation current ramps up during $\sim 300$ ns to its optimum level for gain creation. The presence of the seed laser emission, as shown in Fig. 3, reduces the slave-laser-emission delay from about 550 to $360 \mathrm{~ns}$. Additionally, the slave-laser-emission rise time is reduced by a factor of $\sim 2$, indicating that the small signal gain at the beginning of the laser pulse is about twice higher. This effect is due to the colder active crystal conditions early in the excitation of the slave laser. Quantitative estimation of the peak output power, prevented in Fig. 3 by detector saturation, is presented next.

To obtain output power estimates, the intensity of the two-laser system emission was calibrated using a Ge:Ga detector with small variable input aperture that prevents saturation. A photocurrent of $1.5 \mathrm{~mA}$ was observed using an aperture of $\sim 80 \mu \mathrm{m}$ diameter. According to the $2.2 \mathrm{~A} / \mathrm{W}$ detector sensitivity, this photocurrent corresponds to $0.7 \mathrm{~mW}$ of the absorbed terahertz power. Taking into account the $\sim 20000$ geometrical factor of the aperture relative to the emitted beam cross section and the absorption efficiency factor of $1 / 3$, a peak power of $\sim 40 \mathrm{~W}$ is estimated for the peak output power of the coupled laser. For a laser emission pulse length of $\sim 2-3 \mu \mathrm{s}$, the emission pulse energy estimate is $\sim 100 \mu \mathrm{J}$.

Figure 4 presents stimulated emission for the coupled laser system when the slave-laser excitation pulse is below threshold for different predelays of the seed laser emission pulse. The slave laser excitation high-voltage pulse starts at $1 \mu \mathrm{s}$ as indicated by the arrow, and it ends at the $3 \mu$ s mark on the time axis. The figure demonstrates that injection seeding can force the slave laser to operate even though the applied fields are under the lasing threshold.

Figure 5 shows the signal detected at the end of the light

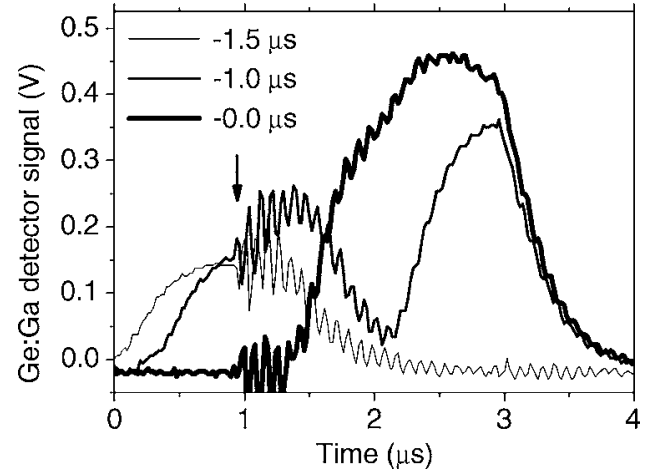

FIG. 4. Effect of injection seeding on subthreshold slave laser. The arrow indicates the onset of slave laser excitation. The legend indicates the trigger advance of the seed laser excitation relative to that of the slave laser.

pipe external to the cryostat by the Golay cell. The lowest trace is the signal from the seed laser alone. The middle trace is from the slave laser, operating alone at the point of its highest gain on total reflection modes with collimated outcoupling via edge bevels. The highest trace corresponds to the coupled two-laser operation. Considering the power estimate above, we infer from the signal ratios of Fig. 5 that the powers of the seed, slave, and synchronized two-laser systems are $0.7,30$, and $40 \mathrm{~W}$, respectively. The result demonstrates that predelayed injection seeding with $0.7 \mathrm{~W}$ provides an increase over the simple superposition of the two lasers by $10 \mathrm{~W}$.

Figure 6 presents spectra of the slave laser emission with and without seed-laser injection. Although the injection is far from spectrally optimized, Fig. 6 demonstrates that injection seeding causes remarkable (several times) increase of the laser emission intensity at 105-112 $\mu \mathrm{m}$. The presence of an emission mode at $117 \mu \mathrm{m}$ with and without the seeding explains why the total rise in intensity is limited to $33 \%$, as found in Fig. 5. We expect a stronger effect of the seeding on the output laser intensity when both lasers are forced to operate at a single frequency by means of efficient intracavity frequency selection. $^{5-9}$

\section{DISCUSSION}

We demonstrated $40 \mathrm{~W}$ output power for dissipated electrical power in the slave laser crystal of order $500 \mathrm{~kW}$. The

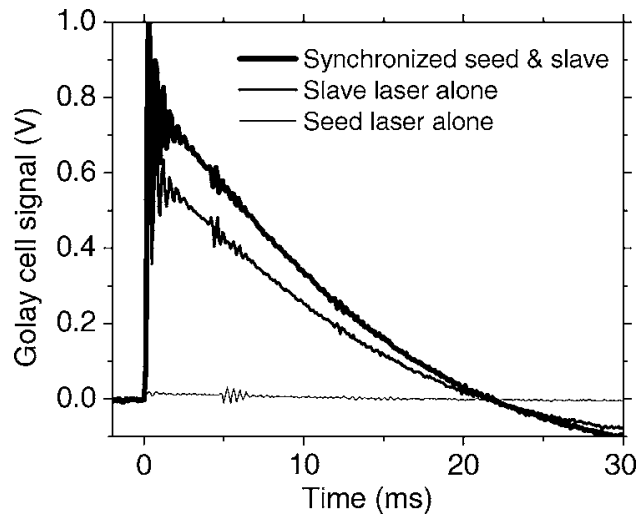

FIG. 5. Laser pulse energies of the seed and slave lasers separately and coupled, recorded by a Golay cell. 


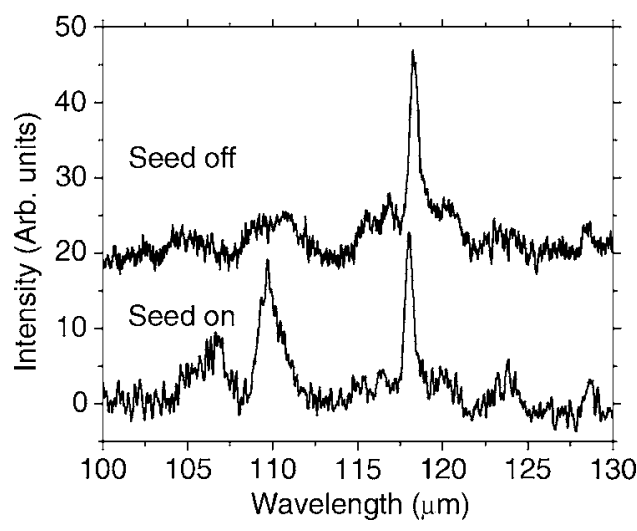

FIG. 6. Spectrum of slave laser emission with and without injection seeding. The latter spectrum is vertically shifted by 20 units for clarity.

claimed theoretical conversion efficiency (optical output/ electrical input) is $10^{-3}-10^{-4}$. 1,3 Thus, the obtained experimental value of $p$-Ge laser efficiency of $10^{-4}$ is in good agreement with the theoretical expectations.

Despite the achieved peak power enhancement, the dual laser system shares the well-known cryogenic requirement of traditional $p$-Ge lasers. A possible solution is delta doping, which the authors have theoretically explored in a number of recent papers. ${ }^{11-15}$ The mechanism of generation of terahertz stimulated emission on direct optical transitions between subbands of the valence band in bulk semiconductors in crossed $E$ and $B$ fields, well known for bulk $p$-Ge lasers, ${ }^{1}$ has several limitations which lead to low efficiency and liquid helium temperature requirements for the laser operation. These limitations are mainly caused by insufficient gain in the face of lattice absorption, which rapidly grows with temperature, and the negative impact of impurity scattering at high doping concentrations. As a possible solution, one might consider the in-plane configuration of the intervalence-band terahertz laser on delta-doped multilayer Ge thin films, ${ }^{12}$ based on spatial separation of light and heavy hole streams, which allows remarkable increase of the gain and the saturation power threshold in comparison with bulk $p$-Ge lasers. Besides increasing the output power, this gain medium is predicted to have sufficient small signal gain coefficient to operate at liquid nitrogen temperatures. Although terahertz quantum cascade lasers have already achieved this temperature milestone, the unique very wide gain spectrum $(2-4 \mathrm{THz})$ for the transitions between valence subbands is potentially important for building a terahertz laser with wide tunability, which none of the other known terahertz semiconductor lasers possesses.

Although the minimum required power for the seed laser is not determined in this work, from the characteristics of the seed laser used here $\sim 100 \mathrm{~mW}$ may suffice. Any other terahertz laser with output of this power in the range 1.5-4.2 THz might be used instead. The seed laser used in this paper may be operated with an intracavity frequency selector to achieve single mode emission ${ }^{5-8}$ or it may be operated in cyclotron resonance mode, which has the benefit of narrow-line magnetically tuned emission ideal for matching the mode spectrum of the large slave laser.

The simplicity and relatively low cost per watt for ger- manium lasers, compared to other terahertz lasers, make the wide dissemination of the technology and a number of unique applications possible. A high peak intensity, high spectral density, cryogenic laser system can play an important role in the laboratory development of new terahertz technologies, especially in characterizing band structure and mechanisms of photoresponse and energy dissipation in semiconductor nanostructures. Traditional $1 \mathrm{~W}$ germanium lasers have already been used to study quantum Hall devices, ${ }^{16}$ subnanosecond dynamics caused by intracavity quantum-well structures, ${ }^{17}$ loss properties of semiconductor interfaces, ${ }^{18}$ amplification by active semiconductor devices, ${ }^{19}$ and modulation of free carrier density within optically thin semiconductor layers. ${ }^{20}$ High-resolution high-contrast terahertz imaging of samples within a cryogenic environment has revealed field/temperature/Landau gap distributions in two dimensional electron gas systems, ${ }^{21}$ and the $p$-Ge laser is a convenient source for such research. The appearance of meV gaps in the band structure of single-wall carbon nanotube field-effect transistors subjected to a magnetic field, modification of Coulomb blockades due to terahertz radiation, and modulation of single-molecule or graphene conductance by intense terahertz fields are all suitable subjects for fundamental and applied research using a high intensity $p$-Ge laser.

In summary, $p$-Ge laser emission with a peak power of order $40 \mathrm{~W}$ was achieved by means of injection seeding of a large active $p$-Ge slave laser operating on total internal reflection modes. We demonstrated that $0.7 \mathrm{~W}$ of injection seeding can increase the output power of the $p$-Ge laser by $10 \mathrm{~W}$, which suggests considerable promise for terahertz $p$-Ge laser systems with distributed active elements. Also it has been demonstrated that the output spectrum of the coupled two-laser system is strongly dependent on the spectrum of the low power seed laser, which makes the use of $p$-Ge laser active crystals as broadband terahertz laser amplifiers possible.

\section{ACKNOWLEDGMENTS}

We acknowledge financial support from US Navy Contract No. N00164-06-C-6022 "High-Power Terahertz Laser SBIR," and technical point of contact of Timothy Bradley. Two of us (A.V.M. and V.N.S.) also thank Russian Foundation for Basic Research for particular support of this work (Grant No. 07-02-01487).

${ }^{1}$ E. Bründermann, in Long Wavelength Infrared Semiconductor Lasers, edited by H. K. Choi (Wiley, Hoboken, NJ, 2004), pp. 279-343.

${ }^{2}$ A. V. Murav'ev, I. M. Nefedov, S. G. Pavlov, and V. N. Shastin, Quantum Electron. 23, 119 (1993)

${ }^{3}$ A. A. Andronov, A. V. Muravjev, I. M. Nefedov, Yu. N. Nozdrin, S. A. Pavlov, V. N. Shastin, Yu. A. Mityagin, V. N. Murzin, S. A. Stoklitsky, I. E. Trofimov, and A. P. Chebotarev, in Proceedings of the 18th International Conference on Physics of Semiconductors, Stockholm, Sweden, 1986, edited by O. Engstrom (World Scientific, Singapore, 1986).

${ }^{4}$ K. Unterrainer, M. Helm, E. Gornik, E. E. Haller, and J. Leotin, Appl. Phys. Lett. 52, 564 (1988).

${ }^{5}$ A. V. Muravjov, S. H. Withers, H. Weidner, R. C. Strijbos, S. G. Pavlov, V. N. Shastin, and R. E. Peale, Appl. Phys. Lett. 76, 1996 (2000).

${ }^{6}$ E. W. Nelson, A. V. Muravjov, S. G. Pavlov, V. N. Shastin, and R. E. Peale, Infrared Phys. Technol. 42, 107 (2001).

${ }^{7}$ T. W. Du Bosq, R. E. Peale, E. W. Nelson, A. V. Muravjov, C. J. Fredrick- 
sen, N. Tache, and D. B. Tanner, J. Appl. Phys. 94, 5474 (2003).

${ }^{8}$ T. W. Du Bosq, R. E. Peale, E. W. Nelson, A. V. Muravjov, D. A. Walters, G. Subramanian, K. B. Sundaram, and C. J. Fredricksen, Opt. Laser Technol. 37, 87 (2005).

${ }^{9}$ A. A. Andronov, V. A. Kozlov, S. A. Pavlov, and S. G. Pavlov, Opt. Quantum Electron. 23, S205 (1991).

${ }^{10} \mathrm{~S}$. Kraft, O. Frenzl, O. Charlier, T. Cronje, R. O. Katterloher, D Rosenthal, U. Grozinger, and J. W. Beeman, Proc. SPIE 4013, 233 (2000)

${ }^{11}$ M. V. Dolguikh, A. V. Muravjov, R. E. Peale, M. Klimov, O. A. Kuznetsov, and E. A. Uskova, J. Appl. Phys. 98, 023107 (2005).

${ }^{12}$ M. V. Dolguikh, A. V. Muravjov, and R. E. Peale, J. Appl. Phys. 99 093106 (2006).

${ }^{13}$ R. E. Peale, M. V. Dolguikh, and A. V. Muravjov, J. Nanoelectron. Optoelectron. 2, 51 (2007).

${ }^{14}$ M. V. Dolguikh, A. V. Muravjov, R. E. Peale, R. A. Soref, D. Bliss, C. Lynch, and D. W. Weyburne, Proc. SPIE 5931, 310 (2005).
${ }^{15}$ M. V. Dolguikh, A. V. Muravjov, and R. E. Peale, Phys. Rev. B 73, 075327 (2006)

${ }^{16}$ C. Stellmach, A. Hirsch, G. Nachtwei, Yu. B. Vasilyev, N. G. Kalugin, and G. Hein, Appl. Phys. Lett. 87, 133504 (2005).

${ }^{17}$ A. V. Muravjov, R. C. Strijbos, C. J. Fredricksen, H. Weidner, W. Trimble, S. H. Withers, S. G. Pavlov, V. N. Shastin, and R. E. Peale, Appl. Phys. Lett. 73, 3037 (1998).

${ }^{18}$ E. W. Nelson, S. H. Withers, A. V. Muravjov, R. C. Strijbos, R. E. Peale, S. G. Pavlov, V. N. Shastin, and C. J. Fredricksen, IEEE J. Quantum Electron. 37, 1525 (2001).

${ }^{19}$ A. V. Muravjov, S. H. Withers, S. G. Pavlov, V. N. Shastin, and R. E. Peale, J. Appl. Phys. 86, 3512 (1999).

${ }^{20}$ C. J. Fredricksen, A. V. Muravjov, and R. E. Peale, Proc. SPIE 5332, 47 (2004).

${ }^{21}$ K. Ikushima, H. Sakuma, S. Komiyama, and K. Hirakawa, Phys. Rev. Lett. 93, 146804 (2004). 